\title{
Modern electronic apex locators are reliable for deter- mining root canal working length
}

\author{
How accurate are electronic apex locators in the determination of root \\ canal length?
}

Haffner C, Folwaczny M, Galler K, Hickel R.

Accuracy of electronic apex locators in comparison to actual length - an in vivo study. J Dent. 2005; 33:619-625

Methods The investigation was carried out by a single operator who was taught to use the different types of electronic apex locators (EAL) according to the operators' manuals. Four EAL were tested: Root ZX (J. Morita Corporation, Tokyo, Japan); Endy (Loser, Leverkusen, Germany); Justy II (Hager-Werken, Duisburg, Germany); and Endox (Lysis, Milan, Italy). Forty root canals were measured prior to tooth extraction for periodontal, endodontic, prosthetic or orthodontic reasons. Canals were rinsed with hydrogen peroxide (3\%) and dried with paper points. K-Files (Dentsply-Maillefer, Konstanz, Germany; ISO sized 15, length $28 \mathrm{~mm}$ ) were used as active electrodes, with the exception of tests with the Endox device, for which special needles are provided by the manufacturer. After extraction and storage in isotonic sodium chloride solution, teeth were sectioned longitudinally until the root canal was longitudinally exposed. Using a stereo microscope, a $15 \mathrm{~K}$-file was inserted into the canal until the tip reached the apical constriction; it was then marked, removed and the length read off a steel rule.

Results Root canal length could be determined for all the teeth using the EAL and the actual root canal length could be determined by microscopic investigation. The Justy II gave the most accurate results, with a mean difference between the actual length of the root canal and the working length of $0.2 \mathrm{~mm}$ [standard deviation (SD), \pm 0.7 ]. This compared with mean differences between measured and actual lengths from the other devices as follows: Root ZX (mean, $0.3 \mathrm{~mm}$; SD, \pm 0.6 ); Endy (mean, $0.7 \mathrm{~mm}$; SD, \pm 1.1 ); Endox (mean, $1.3 \mathrm{~mm}$; SD, \pm 1.7 ). The numbers of samples are shown in Table 1.

Table 1. Samples with exact location of apex and determination of working length to within $\pm 0.5 \mathrm{~mm}$

\begin{tabular}{lllll}
\hline & \multicolumn{2}{c}{ Samples exactly at apex } & \multicolumn{2}{l}{ Samples within $\pm 0.5 \mathrm{~mm}$ of } \\
& $\mathrm{N}$ & Proportion (\%) & $\mathrm{N}$ & Proportion (\%) \\
\hline Root ZX & 18 & 45 & 31 & 78 \\
Endy & 7 & 18 & 27 & 67 \\
Justy II & 18 & 45 & 32 & 80 \\
Endox & 8 & 20 & 13 & 31
\end{tabular}

Conclusions The data revealed different accuracy in working length determination of the four EAL evaluated but, overall, modern EAL offered a reliable method for endodontic working length determination.

Address for correspondence: C Haffner, Department of Restorative Dentistry, Ludwig-Maximilians University, Goethestrasse 70, D-80336 Munich, Germany. E-mail: chaffner@dent.med.uni-muenchen.de.

\section{Commentary}

One problem that clinicians face in endodontics is how to accurately identify and maintain the biological length of the root canal systems. It has been established that the junction of the dentin and the cementum (CDJ) is the landmark that determines the end of this biological length, ${ }^{1,2}$ from a starting point on the coronal side of the tooth (reference point, or area), which must be measured to achieve maximum success during nonsurgical root canal treatment. The variations in anatomy of tooth apices, both by age and tooth type, and the electric properties of each root canal according to its pathological status, ${ }^{3}$ make the task of determining the exact location of the apical constriction all the more challenging.

Methods for studying the accuracy of EAL have varied between invitro, in-vivo and radiographic. The former use electroconductive materials to simulate the clinical situation, alginate, gelatine, agar or saline media all giving predictable results with apex locators when compared with tooth length. ${ }^{4}$ Some of these media can leak through the apical foramen and cause premature readings although, in contrast, some in-vitro experimental models can in fact give greater accuracy than can be achieved clinically.

In-vivo studies are more representative of the true accuracy of a given apex locator. ${ }^{5}$ Studies that use apex locators to find the working length, cement the file in place, extract the tooth and locate the file under magnification in the root canal emulate what happens in clinical practice. When extraction of the tooth has not been possible, studies have used radiographs to verify the canal length. Problems associated with this are the need to work in two dimensions, with anatomic variation and distortions. ${ }^{6}$

This current study addresses which of the chosen EAL work better under random clinical situations, which in turn provides a superior method to the in-vitro assessments. There are, however, several clinical parameters that might have affected the overall accuracy of the study. First, the endodontic file size 15 was used for all tested root canals in vivo, for all types of teeth. The size of the endodontic file should be proportional to the size of the canal for maximum predictability of the measurement process. For example if a no.15 file is inserted into a larger distal canal or palatal canal of a molar it may give inaccurate readings; this could be prevented by using larger file size. Secondly, the authors also did not carry out 'pre-flaring' on the canals tested although several studies have shown that pre-flaring allows working-length files to reach the apical foramen more consistently, in turn increasing the efficacy of the EAL. ${ }^{7}$ Finally, the study did not look into the probability of having a file at the CDJ, even though the EAL would give false readings (long or short) as the file was removed after the in-vivo reading and was re-inserted in-vitro, leading to measurement error.

\section{Practice point}

The study confirms the reliability of the chosen EAL to determine endodontic working length.

\section{Khaled A Balto}

Faculty of Dentistry, King Abdulaziz University, Jiddah, Saudi Arabia 


\section{ENDODONTICS}

1. Grove C. Why canals should be filled to the dentinocemental junction. J Am Dent Assoc 1930; 17:293-296.

2. Schilder H. Filling root canals in three dimensions. Dent Clin N Am 1967; $11: 723$ 744

3. Ricucci D, Langeland K. Apical limit of root canal instrumentation and obturation Part 2. A histological study. Int Endod J 1998; 31:394-409.

4. Czerw RJ, Fulkerson MS, Donnelly JC, Walmann JO. In vitro evaluation of the accuracy of several electronic apex locators. J Endod 1998; 21:572-575.
5. Shabahang S, Goon WW, Gluskin AH. An in vivo evaluation of Root ZX electronic apex locator. J Endod 1996; 22:616-618.

6. Cox VS, Brown Jr CE, Bricker SL, Newton CW. Radiographic interpretation of endodontic file length. Oral Surg Oral Med Oral Pathol 1991; 72:340-344.

7. Ibarrola JL, Chapman BL, Howard JH, Knowles KI, Ludlow MO. Effect of preflaring on Root ZX apex locators. J Endod 1999; 25:625-626.

Evidence-Based Dentistry (2006) 7, 31-32

doi:10.1038/sj.ebd.6400401 